\title{
Fast Spectral Velocity Estimation Using Adaptive Techniques: In-Vivo Results
}

Gran, Fredrik; Jakobsson, Andreas; Udesen, Jesper; Jensen, Jørgen Arendt

Published in:

IEEE Ultrasonics Symposium, 2007

Link to article, DOI:

10.1109/ULTSYM.2007.253

Publication date:

2007

Document Version

Publisher's PDF, also known as Version of record

Link back to DTU Orbit

Citation (APA):

Gran, F., Jakobsson, A., Udesen, J., \& Jensen, J. A. (2007). Fast Spectral Velocity Estimation Using Adaptive Techniques: In-Vivo Results. In IEEE Ultrasonics Symposium, 2007 (pp. 993-996). IEEE.

https://doi.org/10.1109/ULTSYM.2007.253

\section{General rights}

Copyright and moral rights for the publications made accessible in the public portal are retained by the authors and/or other copyright owners and it is a condition of accessing publications that users recognise and abide by the legal requirements associated with these rights.

- Users may download and print one copy of any publication from the public portal for the purpose of private study or research.

- You may not further distribute the material or use it for any profit-making activity or commercial gain

- You may freely distribute the URL identifying the publication in the public portal 


\title{
Fast spectral velocity estimation using adaptive techniques: In-vivo results
}

\author{
Fredrik Gran ${ }^{1}$, Andreas Jakobsson ${ }^{2}$, Jesper Udesen ${ }^{1}$ and Jørgen Arendt Jensen ${ }^{1}$ \\ 1) Center for Fast Ultrasound Imaging, Ørsted•DTU, Bldg. 348, \\ Technical University of Denmark, DK-2800 Kgs. Lyngby, Denmark \\ 2) Department of Electrical engineering, Karlstad University, Sweden
}

\begin{abstract}
Adaptive spectral estimation techniques are known to provide good spectral resolution and contrast even when the observation window $(O W)$ is very short. In this paper two adaptive techniques are tested and compared to the averaged periodogram (Welch) for blood velocity estimation. The Blood Power spectral Capon (BPC) method is based on a standard minimum variance technique adapted to account for both averaging over slowtime and depth. The Blood Amplitude and Phase Estimation technique (BAPES) is based on finding a set of matched filters (one for each velocity component of interest) and filtering the blood process over slow-time and averaging over depth to find the power spectral density estimate. In this paper, the two adaptive methods are explained, and performance is assessed in controlled steady flow experiments and in-vivo measurements.

The three methods were tested on a circulating flow rig with a blood mimicking fluid flowing in the tube. The scanning section is submerged in water to allow ultrasound data acquisition. Data was recorded using a BK8804 linear array transducer and the RASMUS ultrasound scanner. The controlled experiments showed that the OW could be significantly reduced when applying the adaptive methods without compromising spectral resolution or contrast.

The in-vivo data was acquired using a BK8812 transducer. OWs of 128, 64, 32 and 16 slow- time samples were tested. Spectrograms with duration of 2 seconds were generated. Welch's method required 128 samples to give a reasonable spectrogram, whereas the BPC only required 32 samples before the SNR became a limiting factor. The BAPES managed to display the spectrogram with sufficient quality at 16 slow-time samples.
\end{abstract}

\section{INTRODUCTION}

A typical B-mode ultrasound image consists of about 100 image lines, with each line being created by focusing the ultrasound transducer array on a single point on the current line. The backscattered waves are processed with dynamic receive focusing, and, ideally, only objects along the image line are interrogated. The velocity of moving blood can be estimated by imaging the same image line repeatedly. The slow-time signal (sampled with the pulse repetition frequency), for a specific depth, has a center frequency which is proportional to the axial velocity [1]. A common way of estimating the blood velocity is to estimate the power spectral density (PSD) of the slow-time signal. Displaying the PSD as a function of time (the so-called spectrogram) not only visualizes the blood velocity distribution, but also allows the operator to track the timevariations of the blood. The velocity distribution is important for detecting turbulent flow [2], [3] which can be used for diagnosing carotid artery stenosis [4].
In ultrasound imaging, the PSD is normally estimated using an averaged periodogram approach, also known as Welch's method [5], [6]. However, as is well-known, the method suffers from either poor resolution or high leakage, or both, and to achieve sufficient spectral resolution, the duration of the observation window (OW) has to be long. This implies that a large number of transmissions (about 100) has to be carried out to obtain a sufficiently accurate PSD estimate. The following problems emerge:

- The temporal resolution of the spectrogram will be poor due to the long duration of the the averaging kernel used for computing the PSD estimate.

- Interleaved B-mode/Doppler sequences have to be used to update the B-mode image. The sequences can be interleaved so that every second transmission is a B-mode acquisition. This lowers the velocity range by a factor of two. Data can also be acquired in blocks, so that an entire $\mathrm{B}$-mode image is acquired in one sweep. This leaves holes in the spectrogram.

- If the second solution is chosen, due to the lack of Doppler data during B-mode data acquisition, it is necessary to acquire B-mode data as seldom as possible, resulting in poor frame rate on the B-mode images.

A thorough explanation of the problems encountered in spectral Doppler ultrasound can be found in [7]

In this paper, we formulate a data-adaptive blood velocity spectral estimator based on the matched filterbank (MAFI) framework [6], [8]. The purpose is to investigate the possibility of decreasing the OW with maintained spectral resolution and contrast. With a decreased OW, more flexibility would be possible when designing the Doppler/B-mode sequences and all three of the aforementioned problems could potentially be solved.

\section{Methods}

\section{A. Data model}

To estimate the blood velocity at a given location, a number of transmissions are carried out in the same direction. After focusing, the resulting image lines represent a time series over depth. Let $y_{k}(l)$ denote the available (stationary) data sample at slow-time (transmission-number) $l$ and fast-time (proportional to depth) $k$, of which the blood velocity spectrum is to be estimated. For a generic (axial) velocity $v_{z}, y_{k}(l)$ can 
be expressed as [1] (assuming that In-phase and Quadrature channels have been created)

$$
y_{k}(l)=\alpha_{v_{z}} e^{j\left\{k \frac{\omega_{c}}{f_{s}}-l \frac{2 \omega_{c} v_{z}}{c f_{p r f}}\right\}}+w_{k}(l),
$$

for $k=0, \ldots, K-1$ and $l=0, \ldots, \widetilde{L}-1$, where $\alpha_{v_{z}}$ denotes the (complex-valued) amplitude of the sinusoidal signal at velocity $v_{z}$, assumed to be constant over the slow-time OW, and $w_{k}(l)$ is the residual (or noise) term containing the signal components at all velocities different from $v_{z}$. Furthermore, $\omega_{c}=2 \pi f_{c}$, with $f_{c}$ denoting the center frequency of the transducer, $f_{s}$ the sampling frequency of the system, $f_{p r f}$ the pulse repetition frequency, and $c$ the speed of sound. We note that the second term in the exponential in (1) is due to the time shift occurring when the blood particles move between transmissions. The problem of estimating the blood velocity spectral density can thus be expressed as forming an estimate of $\left|\alpha_{v_{z}}\right|^{2}$, for each velocity of interest. For a given depth (fasttime sample) $k$, let

$$
\mathbf{y}_{k}(\ell) \triangleq\left[\begin{array}{lll}
y_{k}(\ell) & \ldots & y_{k}(\ell+N-1)
\end{array}\right]^{T},
$$

for $\ell=0, \ldots, L-1$, with $L=\widetilde{L}-N+1$ representing the total number of slow-time vectors in the time-series. By introducing $\phi=\omega_{c} / f_{s}$ and $\psi=-2 \omega_{c} v_{z} / c f_{p r f}$, (2) can be written as

$$
\mathbf{y}_{k}(\ell)=\alpha_{v_{z}} \mathbf{a}_{\psi} e^{j k \phi+j \ell \psi}+\mathbf{w}_{k}(\ell)
$$

where $\mathbf{w}_{k}(\ell)$ is formed similar to $\mathbf{y}_{k}(\ell)$, and

$$
\mathbf{a}_{\psi} \triangleq\left[\begin{array}{llll}
1 & e^{j \psi} & \ldots & e^{j \psi(N-1)}
\end{array}\right]^{T} .
$$

\section{B. The averaged periodogram (Welch)}

The averaged periodogram is a well known and well used method for estimating the PSD and is given by

$$
\hat{P}_{\text {welch }}(\psi)=\mathbf{a}_{\psi}^{H} \hat{\mathbf{R}} \mathbf{a}_{\psi},
$$

where

$$
\hat{\mathbf{R}}=\frac{1}{K L} \sum_{k=0}^{K-1} \sum_{l=0}^{L-1} \mathbf{y}_{k}(l) \mathbf{y}_{k}^{H}(l),
$$

is the estimate of the auto-correlation matrix of the data.

\section{Blood Spectral Power Capon (BPC)}

The Blood Spectral Power Capon estimate of the PSD is formed by the following optimization problem

$$
\mathbf{h}_{\psi}=\arg \min _{\mathbf{h}_{\psi}} \mathbf{h}_{\psi}^{H} \mathbf{R} \mathbf{h}_{\psi} \quad \text { subject to } \quad \mathbf{h}_{\psi}^{H} \mathbf{a}_{\psi}=1 .
$$

This can be interpreted as finding a filter $\mathbf{h}_{\psi}$, for each frequency of interest, which minimizes the filtered power of the signal, under the constraint that the amplitude of the frequency component of interest, passes through the filter unaffected.

An analytical solution to (7) can be found [6].

$$
\hat{P}_{\mathrm{BPC}}(\psi)=\frac{1}{\mathbf{a}_{\psi}^{H} \hat{\mathbf{R}}^{-1} \mathbf{a}_{\psi}} .
$$

\section{Blood Amplitude and Phase EStimation (BAPES)}

Here, a matched filter bank is used to estimate the PSD of the blood process. The matched filterbank is found by designing a filter (for each frequency of interest) based on the following optimization problem

$$
\mathbf{h}_{\psi}=\arg \min _{\mathbf{h}_{\psi}} \mathbf{h}_{\psi}^{H} \mathbf{Q}_{w} \mathbf{h}_{\psi} \quad \text { subject to } \quad \mathbf{h}_{\psi}^{H} \mathbf{a}_{\psi}=1,
$$

where $\mathbf{Q}_{w}$ is the covariance matrix of the noise and interference term $\mathbf{w}_{k}(l)$. It should be stressed that the matrix $\mathbf{Q}_{w}$, changes as different frequency components $\mathbf{a}_{\psi}$ are probed.

Solving the optimization problem in (9) is not as straightforward as solving (7). This is because no direct estimate of $\mathbf{Q}_{w}$ is available. The suggested solution is to use a "two stage approach", where an initial amplitude estimate of the frequency of interest is found, and used to estimate the matrix $\mathbf{Q}_{w}$. Thereafter, the solution to (9) can be found. The details of this process, however, are out of scope for this paper and the interested reader is referred to [9].

\section{RESULTS}

In this section, we will evaluate the performance of the discussed estimators using both steady state flow measured in a circulating flow rig, and in-vivo measurements on the common carotid artery.

\section{A. Flow rig measurements}

The three methods were tested on a circulating flow rig with a blood mimicking fluid flowing in the tube. The scanning section is submerged in water to allow ultrasound data acquisition. Data was recorded using a BK8804 linear array transducer and the RASMUS system [10]. The $f_{\text {prf }}$ was 1.89 $\mathrm{kHz}$ and the maximum velocity in the tube was $0.05 \mathrm{~m} / \mathrm{s}$. The velocity of the fluid was measured in the tube center. The transmitting aperture consisted of the 16 central elements focused with an F-number of 3.3. The data was processed with dynamic receiving focusing using a Hanning apodization over all 128 receiving elements.

To obtain a reliable reference measurement, a weighted averaged periodogram was used. The OW was chosen to be $N=256$ samples to ensure sufficient spectral resolution. The number of samples used in the averaging was $\tilde{L}=400$. The number of samples used for averaging in the fast-time direction was $K=40$. Prior to PSD estimation, the data was weighted using a Hamming window. 144 independent PSD estimates were created and the mean of these are represented as the light gray plot in Figs. 2 and 1. The reader should be aware that the ultimate goal is to reduce the total number of observations of the blood process $\tilde{L}$. For the results in this section, this is, however, not possible. This is due to the need for a reference spectrogram, which is based on a weighted averaged periodogram, which needs a long averaging kernel to perform adequatly. In the section describing the in-vivo results, however, the averaging kernel will be chosen to depend on the OW, and the benefits from having a shorter OW can be fully exploited.

Next, the three methods described in Section II were tested as a function of OW. Again, 144 PSD estimates were created 
of the flow process. The mean of the PSD estimates for $N=$ 128 can be seen in Fig. 1. $\tilde{L}$ was still chosen to be 400 and $K=40$. All three methods are able to resolve the spectrum of the blood signal. Welch's method displays somewhat more spectral smoothing compared to BPC and BAPES.

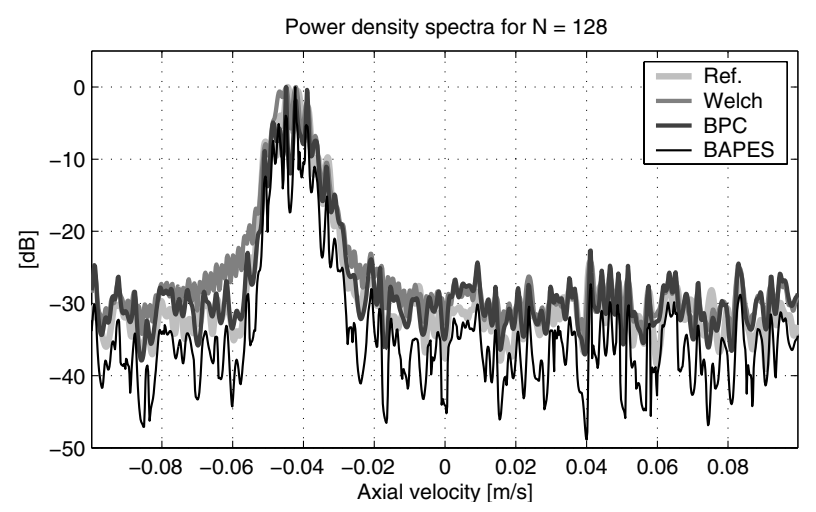

Fig. 1. The mean of the PSD estimates for $N=128, L=400$ and $K=40$. The reference PSD is given in light gray. All three methods are able to resolve the spectrum of the blood signal. Welch's method displays more spectral smoothing compared to BPC and BAPES.

It is well known that for long OWs ${ }^{1}$ Welch's methods has good spectral resolution, so the interesting thing is to investigate the methods performance when $N$ is small. In Fig. 2 , the mean of 144 PSD estimates for $N=4, L=400$ and $K=40$ can be seen. It is obvious that Welch's method fails miserably in finding the PSD. Also the BPC has trouble in finding the PSD and displays one sharp peak instead of the true distribution. Only the BAPES can be considered to resolve the PSD as well as the reference method.

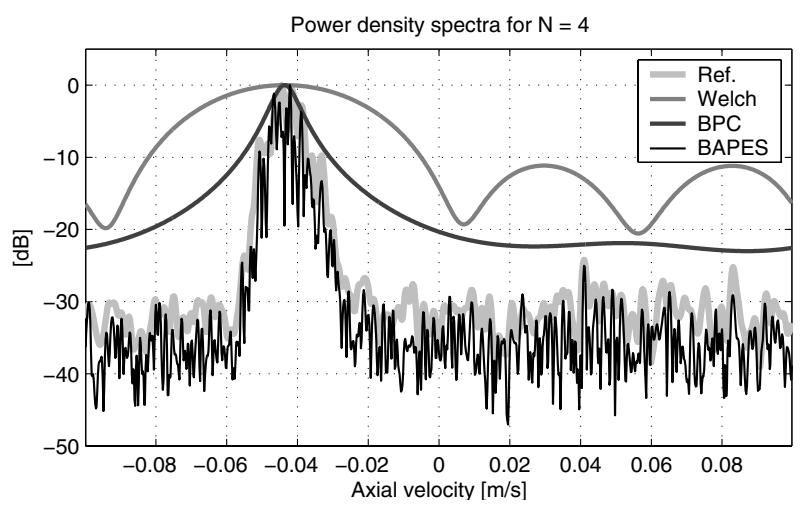

Fig. 2. The mean of the PSD estimates for $N=4, L=400$ and $K=40$. The reference PSD is given in light gray. Welch's method fails miserably in finding the PSD. Also the BPC displays one sharp peak instead of the true distribution. Only the BAPES can be considered to resolve the PSD as well as the reference.

The mean relative bias compared to the maximum of the reference PSD is given by

$$
\mathrm{MRB}=10 \log _{10}\left\{\frac{\frac{1}{P} \sum_{p=0}^{P-1} \bar{P}\left(\psi_{p}\right)-P_{\text {ref }}\left(\psi_{p}\right)}{\max P_{\text {ref }}\left(\psi_{p}\right)}\right\},
$$

${ }^{1}$ In this context, $N=128$ is considered a long OW where $\bar{P}\left(\psi_{p}\right)$ is the mean of the PSD estimate in question, $P_{\text {ref }}\left(\psi_{p}\right)$, is the reference PSD and $P$ is the number of frequency values investigated. The MRB is given in Fig. 3 for the three methods. Welch's method displays worst performance when $N$ is small. Welch's method and the BPC, both improve their MRB as $N$ grows whereas the BAPES has a relatively constant MRB as a function of $N$ showing superior performance at small $N$.

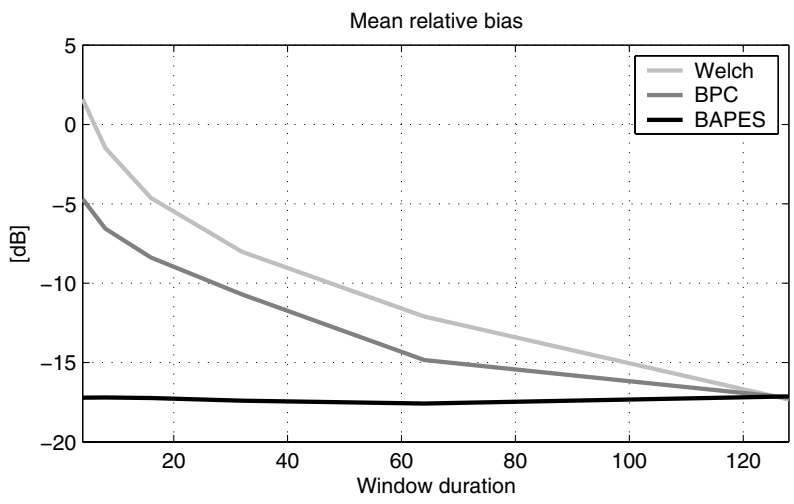

Fig. 3. The MRB for the three methods. BAPES shows the best performance and is not improved as $N$ grows. The BPC is better than Welch's method and improves in terms of $\mathrm{MRB}$ as $N$ grows.

\section{B. Measurements on the common carotid artery}

In-vivo measurements were also carried out. The RASMUS system was used when collecting the data using a BK8812 linear array transducer. The transmitted pulse was a single cycle sinusod at $5 \mathrm{MHz}$ and the $f_{\text {prf }}$ was $12 \mathrm{kHz}$. To make the data narrowband, the received echoes where filtered using an eight cycle sinusoid at $5 \mathrm{MHz}$ before further processing. The common carotid artery of a healthy 36 year old male was scanned by one of the authors. The spectra were plotted as a function of time, so that the full temporal velocity distribution could be visualized. The OW was varied and the spectrograms for $N=128$ and $N=16$ can be seen in Fig. 4 and Fig. 5, respectively.

The number of segments in slow-time used when estimating the covariance matrix was chosen to be $L=N+1$. This was done to achieve the improved time-resolution possible when decreasing the OW. The number of fast-time samples was still $K=20$ and approximately 1.7 seconds of data were generated. The first experiment involved $N=128$ and the result can be viewed in Fig. 4 at a dynamic range of $40 \mathrm{~dB}$. When using a long OW, all three methods are able to show the spectrogram properly. The sidelobes visible in the Welch spectrogram are a result of the rectangular weighting of the data. These could easily be removed using another weighting at the expense of spectral resolution.

Next, a shorter OW was chosen. Here $N=16$ and the result can be seen in Fig. 5 with a dynamic range of 40 $\mathrm{dB}$. The spectral resolution of Welch's methods is much to poor to reproduce the spectrogram correctly. The BPC has sufficient spectral resolution and so has BAPES. BAPES displays slightly better signal to noise ratio than the BPC. 

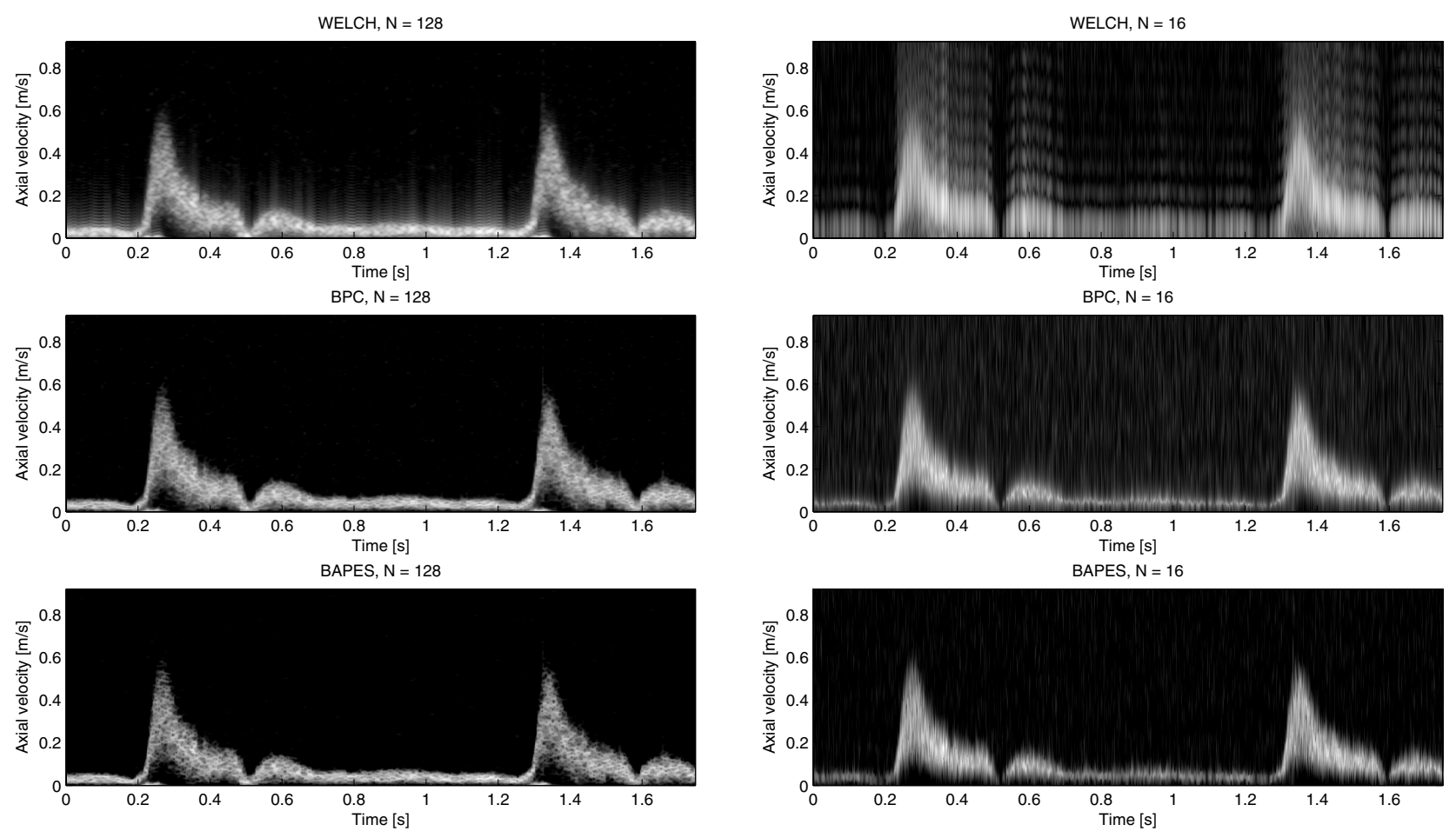

Fig. 4. The spectrogram for the three methods when $N=128$ with a dynamic range of $40 \mathrm{~dB}$. All three methods are able to properly show the spectrogram. The sidelobes visible in the Welch spectrogram are a result of the rectangular weighting of the data. These could easily be removed using another weighting at the expense of spectral resolution.

Observe the finer temporal detail for the BPC and BAPES in the spectrograms in Fig. 5 compared to Fig. 4. This results from the shorter temporal averaging when estimating the spectrogram.

\section{CONCLUSION}

In this paper, we have tested two data-adaptive blood velocity spectral estimators (BPC and BAPES). The two methods were compared to the averaged periodogram (Welch's method) via flow measurements in a circulating flow rig and in-vivo measurements on the carotid artery. It has been shown that, by using the adaptive methods the OW used for estimating the spectrogram can be significantly decreased with maintained spectral resolution. This offers more flexibility when acquiring spectral Doppler data and B-mode data simultaneously. Therefore, the frame rate of the B-mode images could potentially be increased. Furthermore, the time resolution of the spectrogram will increase, offering finer temporal details to be studied.

\section{ACKNOWLEDGMENT}

This work was supported by grant 274-05-0327 from the Danish Research Agency, the Radio-parts foundation and by B-K Medical Aps, Denmark.

\section{REFERENCES}

[1] J. A. Jensen, Estimation of Blood Velocities Using Ultrasound: A Signal Processing Approach, Cambridge University Press, New York, 1996.

Fig. 5. The spectrogram for the three methods when $N=16$ with a dynamic range of $40 \mathrm{~dB}$. The spectral resolution of Welch's methods is here much to poor to reproduce the spectrogram correctly. The BPC has sufficient spectral resolution and so has BAPES. BAPES displays slightly better signal to noise ratio than the BPC.

[2] P. S. Green, "Spectral broadening of acoustic reverberation in Dopplershift fluid flowmeters," J. Acoust. Soc. Am., vol. 36, pp. 1383-1390, 1964.

[3] B. Sigel, R. J. Gibson, K. V. Amatneek, W. R. J. Felix, and A. L. Edelstein, "A doppler ultrasound method for distinguishing laminar from turbulent flow. A preliminary report.," J. Surg. Res., vol. 10, pp. 221224, 1970.

[4] R. W. J. Felix, B. Sigel, R. J. Gibson, J. Williams, and G. L. Popgy, "Pulsed doppler ultrasound detection of flow disturbances in arteriosclerosis," J. Clin. Ultrasound, vol. 4, pp. 275-282, 1976.

[5] P. D. Welch, "The use of fast Fourier transform for the estimation of power spectra: A method based on time averaging over short, modified periodograms," IEEE Trans. Au. Electroacous., vol. AU-15, pp. 70-73, 1967.

[6] P. Stoica and R. Moses, Spectral Analysis of Signals, Prentice Hall, Upper Saddle River, N.J., 2005.

[7] J. A. Jensen, "Spectral velocity estimation in ultrasound using sparse data sets," J. Acoust. Soc. Am., vol. vol 120(1), pp. 211-220, 2006.

[8] P. Stoica, A. Jakobsson, and J. Li, "Matched-Filterbank Interpretation of Some Spectral Estimators," Signal Processing, vol. 66, no. 1, pp. 45-59, April 1998

[9] F. Gran, A. Jakobsson, and J. A. Jensen, "Adaptive blood velocity estimation in medical ultrasound," in IEEE International Conference on Acoustics, Speech and Signal Processing, 2007, vol. 1, pp. 293-296.

[10] J. A. Jensen, O. Holm, L. J. Jensen, H. Bendsen, S. I. Nikolov, B. G. Tomov, P. Munk, M. Hansen, K. Salomonsen, J. Hansen, K. Gormsen, H. M. Pedersen, and K. L. Gammelmark, "Ultrasound research scanner for real-time synthetic aperture image acquisition," IEEE Trans. Ultrason., Ferroelec., Freq. Contr., vol. 52 (5), May 2005. 